\title{
Epitaxial growth of FCC metals on various crystallographic surfaces of $\mathrm{NaCl}$
}

Nilabh Dish ${ }^{1}$ A. Satyaprasad ${ }^{2}$ and Abhay Gautam ${ }^{1}$

${ }^{1}$ Materials Engineering, Indian Institute of Technology, Gandhinagar, Gujarat, India, ${ }^{2}$ Institute for Plasma Research, Gandhinagar, Gujarat, India

Interest in epitaxially grown thin films has seen an exponential growth over the decades due to their significant scientific and technological impact. Techniques combining various substrates and vapor deposition techniques have been used to grow a range of crystallographically oriented thin films. One of the earlier approaches involved the use of freshly cleaved (100) surface of a single crystal $\mathrm{NaCl}$ to grow (100) oriented films of various metals through physical vapor deposition process. This offers a very efficient and convenient method of producing free-standing, oriented single-crystal metal films.

In this work we present a systematic study involving deposition of gold, silver and copper on near (100), (110), (111), and (211) surfaces of $\mathrm{NaCl}$ crystal. These surfaces were prepared using steel angle blocks prepared with CNC machine to polish at appropriate angle. These polishing blocks were designed to use the easy cleave (100) surface of the $\mathrm{NaCl}$ as a reference to polish at appropriate angle to achieve near (110), (111), and (211) surfaces while (100) surface was obtained by usual cleaving technique. Final mechanical polishing for each of these surfaces was done on a $\mathrm{P} 4000$ mesh SiC paper. This was followed by three different surface preparations. First involved polishing the prepared surface on a glass surface using ethanol second was prepared by polishing in distilled water on glass and third surface was prepared by furnace annealing the prepared surface at $760{ }^{\circ} \mathrm{C}$ in air. Each of these surfaces was then used to deposit high purity gold, silver and copper from resistively heated tungsten boat at a base pressure of $\sim 10^{-6}$ torr. The deposition was done by flash evaporation of predetermined amount of material to achieve thinnest continuous metal film. Multiple depositions with estimated thickness ranging between $\sim 40-130 \mathrm{~nm}$ were prepared. These films were then released from salt crystal in deionized water and transferred on to TEM grids for further characterization.

Electron diffraction patterns obtained from these metal films clearly showed that all three metals preferred to grow along similar crystallographic plane as the $\mathrm{NaCl}$ surface. Additionally, the final step of polishing surface with ethanol or water clearly had an effect on the epitaxial growth process with water treated surface showing far fewer randomly oriented crystallites compared to films grown on ethanol treated surface. Effect of water treatment on (100) $\mathrm{NaCl}$ surface has been studied [1] suggesting that surface-adsorption of water-vapor prior to vapor-deposition allows the rapid recrystallization of primarily unordered atomic layers on the cleavage face of $\mathrm{NaCl}$ hence promoting better epitaxy. While another study suggests [2] formation of surface hydroxide during water-vapor treatment of $\mathrm{NaCl}$ surface resulting in epitaxial growth of gold. While growth of (100) and to a limited extent (110) and (111) films of fcc metal on respective $\mathrm{NaCl}$ surface have been reported before but higher index epitaxial growth such as (211) has not been observed earlier.

Compared to water and ethanol treated surfaces, the furnace annealed surface gave the best epitaxy with lowest defect density and fewer randomly oriented crystallites. Fig 1 below a series of bright field (BF) images of Au, $\mathrm{Ag}$, and $\mathrm{Cu}$ grown on annealed (110), (111) and (211) surfaces of $\mathrm{NaCl}$ along with conventional technique of deposition on freshly cleaved (100) surface. 
These results clearly demonstrate that current approach of mechanical polishing of $\mathrm{NaCl}$ crystal near specific crystallographic surface followed by annealing of the surface can be used to prepare epitaxial films along higher index planes of $\mathrm{NaCl}$.

\section{Acknowledgements}

The authors would like to acknowledge the financial support provided by Science and Engineering Research Board (S.E.R.B.) through EMR grant (SB/S3/ME/044/2015).

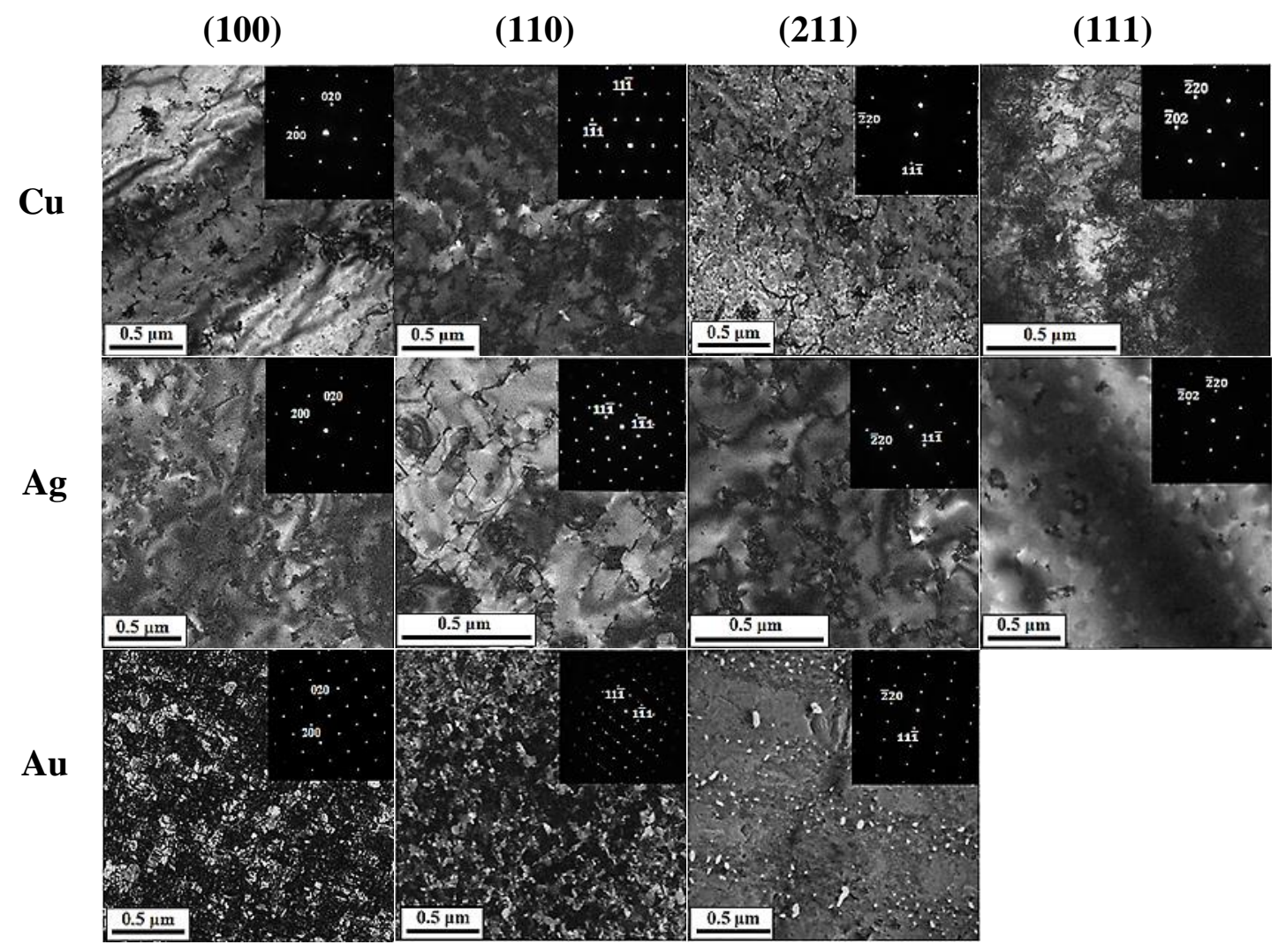

Figure 1.Bright-Field images of free standing films of $\mathrm{Cu}, \mathrm{Ag}$, and $\mathrm{Au}$ grown on annealed (110), (111), and (211) surface and freshly cleaved (100) surface of $\mathrm{NaCl}$ single crystal.

\section{References}

[1] L.E. Murr, M.C. Inman, Effects of vacuum environment on the sub-structure of evaporated F.C.C. metal films, Philos. Mag. 14 (1966) 135-153. https://doi.org/10.1080/14786436608218996.

[2] J.S. Vermaak, C.A.O. Henning, The role of the substrate surface layer in the process of epitaxy part I. The growth of gold films on rocksalt and its substitutional surfaces, Philos. Mag. 22 (1970) 269-280. https://doi.org/10.1080/14786437008228223. 